\title{
Effects of cognitive task on gait initiation in Parkinson disease: Evidence of motor prioritization?
}

\author{
Joe R. Nocera, PhD; ${ }^{1 *}$ Ryan Roemmich; ${ }^{2}$ Jonathan Elrod; ${ }^{2}$ Lori J. P. Altmann, PhD; ${ }^{3}$ Chris J. Hass, PhD ${ }^{2}$ \\ ${ }^{1}$ Department of Veterans Affairs (VA) Rehabilitation Research and Development Center of Excellence, Atlanta VA \\ Medical Center, Decatur, GA; and Department of Neurology, Emory University, Decatur, GA; Departments of \\ ${ }^{2}$ Applied Physiology and Kinesiology and ${ }^{3}$ Speech, Language, and Hearing Sciences, University of Florida, Gaines- \\ ville, $F L$
}

\begin{abstract}
While much is known about the effects of dual tasking on cyclical and continuous motor performance (e.g., locomotion), there is a paucity of information on the effect of dual tasking on the initiation of movement. Therefore, the purpose of this study was to investigate the effect of a concurrent cognitive task on gait initiation in three groups: patients with Parkinson disease, healthy older adults, and healthy young adults. We examined the anticipatory postural adjustment displacements and velocities during single-task gait initiation as well as two dual-task conditions: (1) 0-back + gait initiation and (2) 2-back + gait initiation. The Parkinson disease group exhibited less anticipatory postural adjustment displacement and velocity than their aged-matched healthy peers and young adults during the single- and dual-task gait initiation settings $(p<0.05)$. Of interest was the finding of no additional effect on anticipatory postural adjustment displacement or velocity of gait initiation during the dual-task conditions in any group, including the Parkinson disease group. More traditionally studied gait/balance dual-task paradigms have demonstrated both motor and cognitive decline. Therefore, our results may suggest a prioritization of more "intentional” movement task (e.g., gait initiation) while dual tasking in Parkinson disease.
\end{abstract}

Key words: aging, cognition, dual task, gait initiation, locomotion, $n$-back, neurological disease, Parkinson disease, physical function, prioritization.

\section{INTRODUCTION}

Individuals with Parkinson disease (PD) demonstrate motor impairments during performance of many daily functional tasks, including gait initiation (GI) [1-2]. During GI, patients with PD demonstrate reduced propulsive force generation, increased step length variability, and diminished anticipatory postural adjustments (APA) [1-3]. Collectively, these impairments compromise the necessary momentum production required for efficient and safe GI.

Cognitive decline that accompanies PD is also a critical contributing factor to worsening physical function [4]. Indeed, impaired executive function and attention are related to postural instability in PD [5]. Further, "dual tasking," or the simultaneous performance of motor and/or cognitive tasks, compounds decrements in motor and cognitive performance [6-8]. For example, patients with PD have demonstrated profound effects of secondary cognitive tasks during locomotion such that their ability to

\footnotetext{
Abbreviations: ANOVA = analysis of variance, APA = anticipatory postural adjustments, GI = gait initiation, $\mathrm{H} \& \mathrm{Y}=$ Hoehn \& Yahr, HOA = healthy older adults, HYA = healthy young adults, MANOVA = multivariate analysis of variance, $\mathrm{MMSE}=$ Mini-Mental State Examination, PD = Parkinson disease.

*Address all correspondence to Joe R. Nocera, PhD; Atlanta VA Medical Center, 1670 Clairmont Rd, MS 151R, Decatur, GA 30033. Email: joenocera@emory.edu

http://dx.doi.org/10.1682/JRRD.2012.06.0114
} 
maintain a stable walk is impaired [7].

While much is known about the effects of dual tasking on cyclical and continuous motor performance (e.g., locomotion), there is a paucity of information on the effect of dual tasks on the initiation of movement such as GI. In fact, to our knowledge, only one study exists examining the effect of a cognitive task on GI [9]. This study demonstrated a slowed "response time" to a secondary cognitive task during GI. However, it did not present findings on the displacement and velocity of the APA during GI and it was only specific to healthy young and old adults. The fact that little attention has been devoted to examining GI during PD dual tasking is surprising considering the noted impairments during dual tasks as well as the difficulties patients have initiating transitional movements (e.g., freezing during GI).

For this project, we studied the effects of a concurrent cognitive task on the APA during GI. The APA serve to accelerate the body's center of mass forward and laterally toward the stance foot by moving the center of pressure posterior and toward the stepping leg $[1-2,10]$. Measurement of the displacement of the center of pressure during the APA of GI is a common measure of postural stability, providing insight into the generation of forward momentum needed for locomotion [1-2]. Importantly, we have previously shown that the outcomes of the APA during GI are vulnerable to changes that occur in the system with advanced age and disability [1-2]. Because the APA of GI are thought to be initiated via juxtapositional lobule cortex (formally the supplementary motor area) [11], it is possible that dual tasking during GI will differentially affect motor/cognitive performance because both the motor and cognitive tasks in this paradigm will rely on higher cortical function.

To evaluate the influence of cognitive loading on GI, we utilized an $n$-back task. The $n$-back task requires participants to monitor a sequence of letters and respond whether the current item is the same as the item $n$ positions before. This is a commonly utilized working memory task because accuracy depends on maintaining and updating task-relevant stimuli and inhibiting interference from irrelevant stimuli held in working memory as the trials proceed [12-13]. The working memory load increases as the demands for maintenance, updating, and inhibition increase with the size of $n$. The $n$-back task reliably produces activation in working memory-related cortical regions, namely the prefrontal cortex [13]. Deficits in working memory are commonly implicated in the cognitive pathology of PD [14].
We predicted that both healthy young adults (HYA) and healthy older adults (HOA) would have more robust measures of the APA during GI than PD participants in the absence of the cognitive task, i.e., "single-task GI.” Additionally, we predicted that a greater dual-task effect would be observed in the PD participants, hypothesizing that the decrements in single-task GI performance demonstrated in PD would be exacerbated with the additional requirement of the cognitive task. To test these hypotheses, in addition to single-task GI, we measured the participants' GI performance while they simultaneously performed a 0-back (low working memory load) and a 2-back (high working memory load) task. To further understand the effects of the dual-task paradigm, we calculated the percent correct of 0 - and 2-back performance in both single- and dual-task settings. We hypothesized that as $n$-back complexity increased from 0 to 2 , the PD group would produce greater errors in both single- and dual-task settings.

\section{METHODS}

We examined a total of 37 adults from the greater University of Florida area. Thirteen participants (aged $65.8 \pm 2.0 \mathrm{yr}$ ) were diagnosed as having idiopathic PD by a movement disorders neurologist using standard diagnostic criteria (UK Brain Bank Criteria for PD). Hoehn \& Yahr (H\&Y) disability staging ranged between 1 and 2.5. PD participants were on stable doses of dopaminergics, and evaluations were conducted while the patients were clinically "on," or fully responding to their PD medications. At the time of testing, none of the patients exhibited any dyskinesia, dystonia, or other signs of involuntary movement. Further, PD participants completed the experimental trials without incident or other disruption, such as festination or freezing.

Thirteen HOA (aged $66.4 \pm 2.6 \mathrm{yr}$ ) who were age(1.5 yr) and sex-matched to the PD participants also volunteered. Lastly, 11 sex-matched HYA (aged $20.9 \pm$ $2.1 \mathrm{yr}$ ) participated. These HOA and HYA were free of any acute or chronic condition that would interfere with their participation in the study.

All participants were community living and free of visual impairments that would limit their ability to perceive the visually presented $n$-backs. Further, no participants demonstrated overt cognitive impairment, as measured by a Mini-Mental State Examination (MMSE) score $<26$. 


\section{Instrumentation: Center of Pressure Displacement}

To determine the APA, we determined the displacement and velocity of the center of pressure from ground reaction forces recorded from a force plate (Type 4060 10, Bertec Corp; Columbus, Ohio) embedded level with the floor. Forces and torques were sampled at $360 \mathrm{~Hz}$, filtered with a fourth-order Butterworth low-pass filter with a cutoff frequency of $10 \mathrm{~Hz}$.

\section{Experimental Protocol}

The data collection consisted of three single-task GI trials, three 0-back GI trials, and three 2-back GI trials. Thus, nine experimental GI trials were collected. The order of all GI trials was randomized. During practice trials (2-3), the preferred leg to initiate gait was observed, recorded, and held consistent throughout the data collection. Initial positioning of the feet was self-selected and then subsequently marked to ensure consistent foot placement and stance width throughout the experimental protocol.

\section{Single-Task Gait Initiation}

Participants began each trial standing quietly on the force plate in a relaxed posture and were instructed to distribute weight equally between their two feet and to fix their gaze on a target (fixation cross) at eye level $6 \mathrm{~m}$ in front of them. In response to a visual cue (an illuminated light within the fixation cross), the participants initiated gait and continued to walk the length of the $5 \mathrm{~m}$ walkway.

\section{Dual Task: Gait Initiation and n-Backs}

While standing on the force plate just described, participants were instructed to respond "yes" if the letter was the same as $n$ letters before or "no" if the letter was different. The $n$-back stimulus consisted of 25 letters, of which 20 percent were a "target" stimuli requiring a "yes" response. For the remaining 80 percent of letter stimuli, participants needed to respond "no" for a correct response. Participants were instructed that a failure to respond would be counted as incorrect. The letters were projected on a large screen at eye level $6 \mathrm{~m}$ in front of the standing participant in a fixed location. Each letter was presented on the same screen and space as the fixation cross to minimize the participants having to move their eyes. Each letter was visible for $1.5 \mathrm{~s}$, followed by a $1.5 \mathrm{~s}$ interstimulus interval. During each $n$-back block, at a random interstimulus interval (held consistent for each participant), the illuminated light (same as in the single GI condition) would cue the participants to initiate gait. Importantly, the illuminated cue appeared in the same space on the screen as the fixation cross and letters to minimize eye movement. Participants were instructed to initiate gait after the light signal, continue walking the length of the $5 \mathrm{~m}$ walkway, and wait, if necessary, while continuing to answer "yes" or "no" until the completion of the $n$-back (a blue screen). Similar to previous dualtask paradigms, participants were instructed to perform as accurately as possible and to equally divide their attention between the cognitive and motor tasks [15].

\section{Single Task: n-Backs}

The single-task $n$-back trials were conducted at the same rate as the dual-task condition. However, during the single-task $n$-backs, participants were seated in a quiet room and the $n$-backs were presented on a computer screen. The order of single versus dual task was randomized. To control for practice effects, all participants performed three practice blocks at each level and reported task comprehension.

\section{Landmarks and Data Analysis}

Custom MATLAB software (version 7.9.0, R2009b, The MathWorks; Natick, Massachusetts) was used for identification of the APA. The APA phase began with the initial movement of the center of pressure and ended when the center of pressure was located in its most posterior and lateral position toward the swing limb. Four dependent variables were computed during the APA phase: displacement and velocity of the center of pressure in the anterior-posterior direction and displacement and velocity in the medial-lateral direction toward the swing foot.

The percentage of correct responses during the $n$ backs in the single- and dual-task settings was calculated and averaged across each of the three blocks within each condition, yielding a single percent correct score for single-task 0-back, single-task 2-back, dual-task 0-back, and dual-task 2-back.

A 3 (group) $\times 3$ (condition) repeated measures multivariate analysis of variance (MANOVA) was used to test the overall group (HYA, HOA, PD) differences across conditions (single GI, 0-back GI, 2-back-GI) on the four dependent variables of the APA phase of GI (displacement in the anterior-posterior and medial-lateral directions as well as velocity in the anterior-posterior and medial-lateral 
directions). Additionally, two separate 3 (group) $\times 2$ (condition) repeated measures analyses of variance (ANOVAs) were performed to test the overall group differences across conditions (single 0 -back vs dual 0-back and single 2-back vs dual 2-back) on the percentage of correct responses during the $n$-backs.

\section{RESULTS}

Descriptive statistics (mean and standard deviation) were calculated for all participants on age, education, and MMSE (Table). ANOVA of these measures revealed no significant differences between groups $(p>0.05)$ apart from an obvious difference in age between HYA and the other two groups.

\section{Single- Versus Dual-Task Gait Initiation}

The $3 \times 3$ MANOVA revealed a significant main effect of group, $F(8,62)=2.38, p=0.03$. The PD group demonstrated decreased displacements and velocities compared with the HYA group on all four variables $(p<$ 0.05) (Figure 1). Further, compared with HOA, those with PD demonstrated significantly less displacement in the medial-lateral direction and significantly slower velocity in the anterior-posterior and medial-lateral directions of the APA during GI $(p<0.05)$ (Figure 1). The main effect for condition was not significant, indicating that among the four outcome variables, no significant effect of the dual task was observed within any group (Figure 2). Additionally, the group-by-condition interaction was not significant.

\section{Single- Versus Dual-Task $\boldsymbol{n}$-Backs}

The groups did not statistically differ in the number of correct responses in the single- or dual-task 0-back $(p>0.05)$. The PD group was significantly less accurate during the single-task 2-back than HYA and HOA ( $p=$ 0.01 and $p=0.02$, respectively). Additionally, this effect was exaggerated in the dual-task 2-back. In fact, a post hoc $t$-test comparing PD 2-back performance to chance (50\%) during the dual-task condition (53\%) confirmed no statistical difference. In contrast, the HYA and HOA demonstrated no effect of the dual task on performance of the 2-back (Figure 3).

\section{DISCUSSION}

This study examined GI performance in HYA, HOA, and patients with PD under single-task and two dual-task conditions of varying cognitive load. We hypothesized that both HYA and HOA would have more robust measures of the APA during GI than the PD participants in the absence of the cognitive task, i.e., single-task GI. Additionally, we

Table.

Subject characteristics: sex, age, years of formal education, and Mini-Mental State Examination (MMSE) for healthy young adults (HYA), healthy older adults (HOA), and participants with Parkinson disease (PD), as well Hoehn \& Yahr disability score (H\&Y) and Unified Parkinson Disease Rating Scale (UPDRS) motor and total scores for participants with PD. Data shown as mean \pm standard deviation. Analysis of variance revealed no significant differences between groups apart from obvious age difference between HYA and other two groups.

\begin{tabular}{|c|c|c|c|c|c|c|c|}
\hline \multirow{2}{*}{ Group } & \multirow{2}{*}{$n$} & \multirow{2}{*}{$\begin{array}{l}\text { Age } \\
\text { (yr) }\end{array}$} & \multirow{2}{*}{$\begin{array}{c}\text { Education } \\
\text { (yr) }\end{array}$} & \multirow{2}{*}{ MMSE } & \multirow{2}{*}{ H\&Y } & \multicolumn{2}{|c|}{ UPDRS } \\
\hline & & & & & & Total & Motor \\
\hline \multicolumn{8}{|l|}{$\overline{\mathrm{HYA}}$} \\
\hline Female & 6 & $20.1 \pm 2.7$ & $16.3 \pm 2.5$ & $29.8 \pm 0.4$ & - & - & - \\
\hline Male & 5 & $19.4 \pm 0.9$ & $14.7 \pm 1.0$ & $29.8 \pm 0.4$ & - & - & - \\
\hline Total & 11 & $20.9 \pm 2.1^{*}$ & $15.6 \pm 2.3$ & $29.8 \pm 0.4$ & - & - & - \\
\hline \multicolumn{8}{|l|}{ HOA } \\
\hline Female & 8 & $66.4 \pm 1.8$ & $16.5 \pm 1.5$ & $29.0 \pm 0.8$ & - & - & - \\
\hline Male & 5 & $66.4 \pm 3.5$ & $19.0 \pm 1.8$ & $29.0 \pm 0$ & - & - & - \\
\hline Total & 13 & $66.4 \pm 2.6$ & $18.5 \pm 2.0$ & $29.0 \pm 0.5$ & - & - & - \\
\hline \multicolumn{8}{|l|}{ PD } \\
\hline Female & 8 & $65.4 \pm 1.3$ & $19.5 \pm 1.5$ & $29.0 \pm 0.8$ & $1.9 \pm 0.5$ & $39.5 \pm 3.1$ & $20.8 \pm 3.2$ \\
\hline Male & 5 & $66.4 \pm 2.9$ & $17.0 \pm 2.2$ & $27.6 \pm 1.5$ & $2.0 \pm 0.6$ & $41.1 \pm 2.1$ & $21.3 \pm 2.2$ \\
\hline Total & 13 & $65.8 \pm 2.0$ & $18.0 \pm 2.3$ & $28.5 \pm 1.3$ & $1.9 \pm 0.5$ & $40.3 \pm 2.6$ & $21.0 \pm 2.7$ \\
\hline
\end{tabular}


(a)

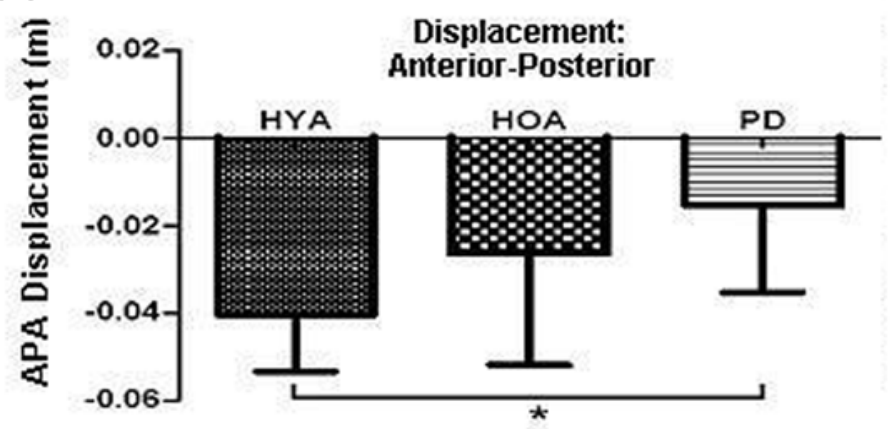

(c)

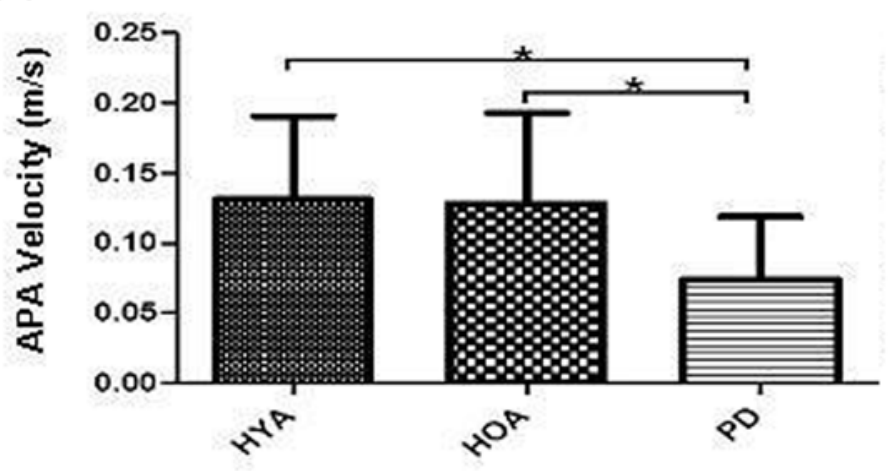

(b)

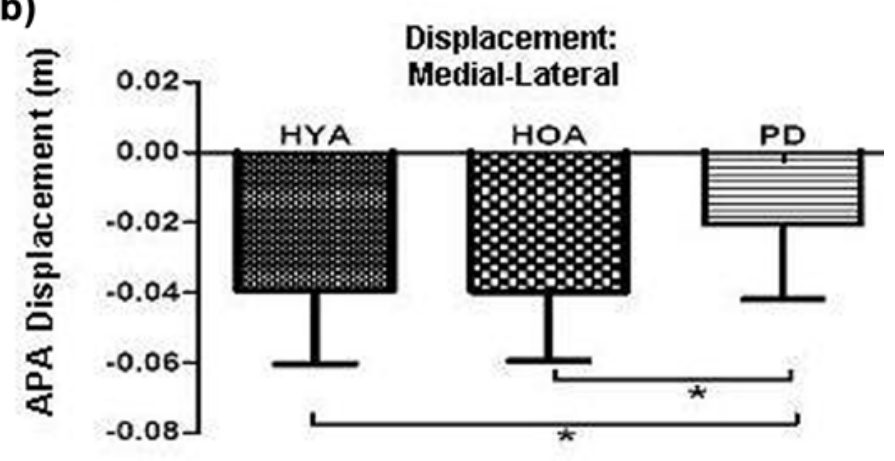

(d)

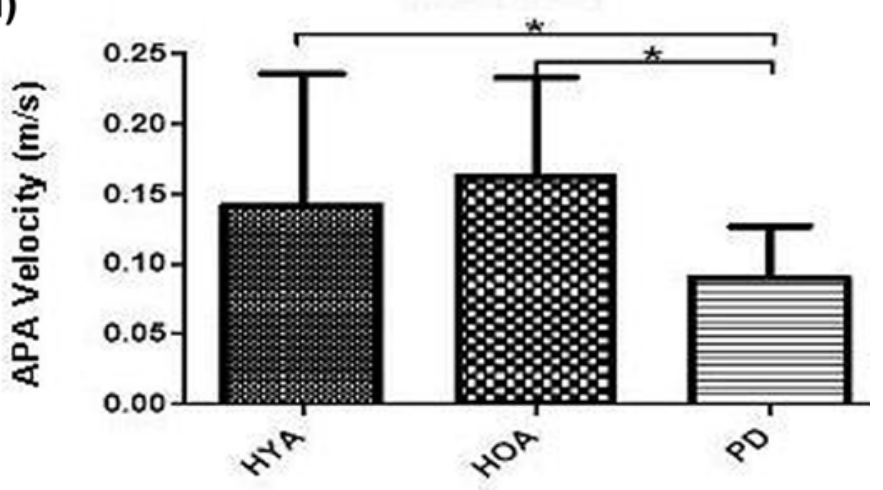

Figure 1.

Mean and standard error values of anticipatory postural adjustments (APA) for healthy young adults (HYA), healthy older adults (HOA), and individuals with Parkinson disease (PD). APA displacement during gait initiation (GI) in (a) anterior-posterior (AP) and (b) medial-lateral (ML) directions. APA velocity during GI in (c) AP and (d) ML directions. Note that PD group demonstrated decreased displacements and velocities when compared with HYA on all four variables $(p<0.05)$. Additionally, when compared with HOA, PD group demonstrated significantly less displacement in ML direction and significantly less velocity in AP and ML directions of APA during GI $(p<0.05)$. *Significant at $p<0.05$.

hypothesized that the decrements in single-task GI performance demonstrated in PD would be exacerbated with the additional requirement of a cognitive load (0- and 2backs). In line with our hypothesis, when we examined the APA phase of GI during both a single- and dual-task paradigm, the PD group exhibited less displacement and velocity than HYA and HOA (Figure 1). Of interest and in contrast to our hypothesis was the finding that the displacement and velocity APA phase of GI was not influenced by the dual-task conditions in the PD group (Figure 2(a)-(d)). Significantly, however, the PD group committed more errors on the 2-back during the dual-task condition (53\% correct) than during the single-task condition (92\% correct) (Figure 3). Of note, the PD participants were early on in the disease (H\&Y 1-2.5), were highly educated (18+ yr of education), and demonstrated no overt cognitive impairment as measured by the MMSE $(28.5 \pm 1.5)$. Considering these factors, the degree to which the PD participants' performance degraded on the 2-back during the simultaneous motor/high-cognitive task condition was quite surprising. In fact, the PD group performed at the level of chance on the 2-back during the dual-task condition. It is important to note this finding may reflect an imbalance in the allocation or a prioritization of motor versus cognitive performance, despite instructions regarding attention allocation.

Posterior center of pressure displacement of the APA phase during GI is vital to generate the forward momentum 
(a)

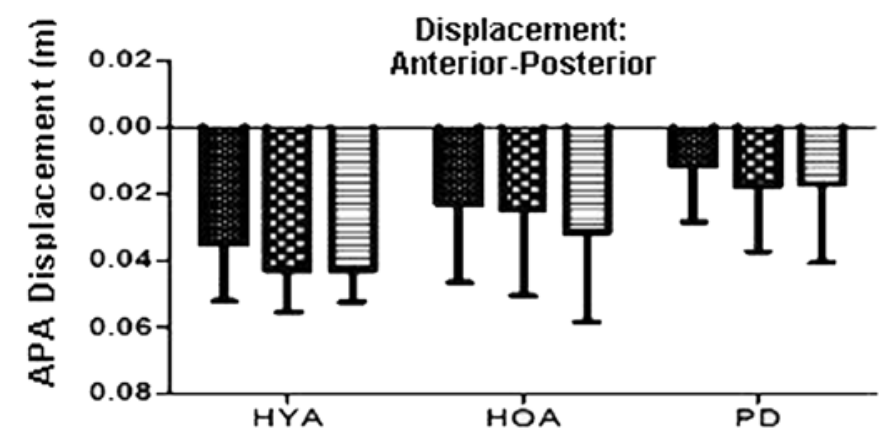

(c)

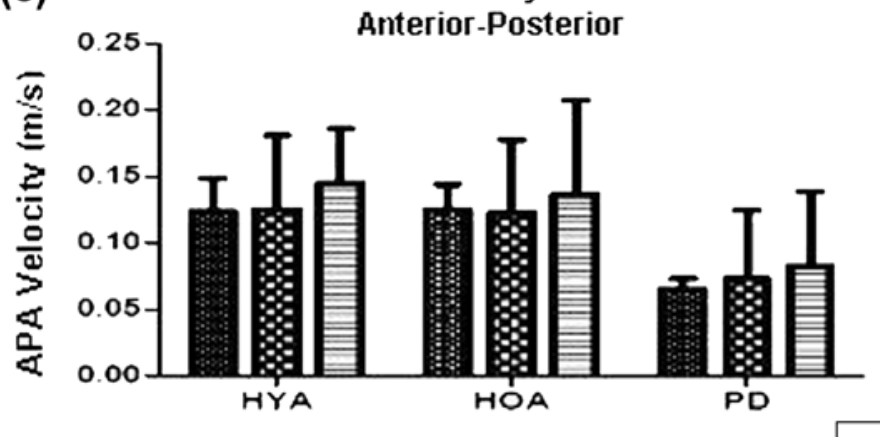

(b)

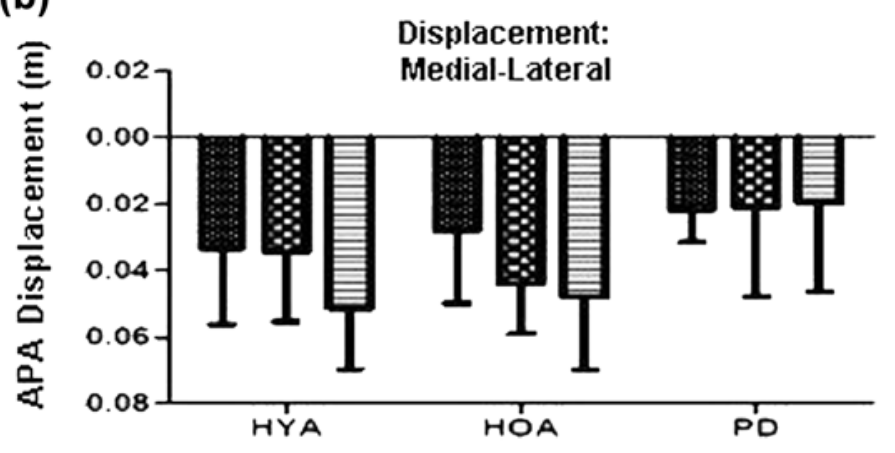

(d)

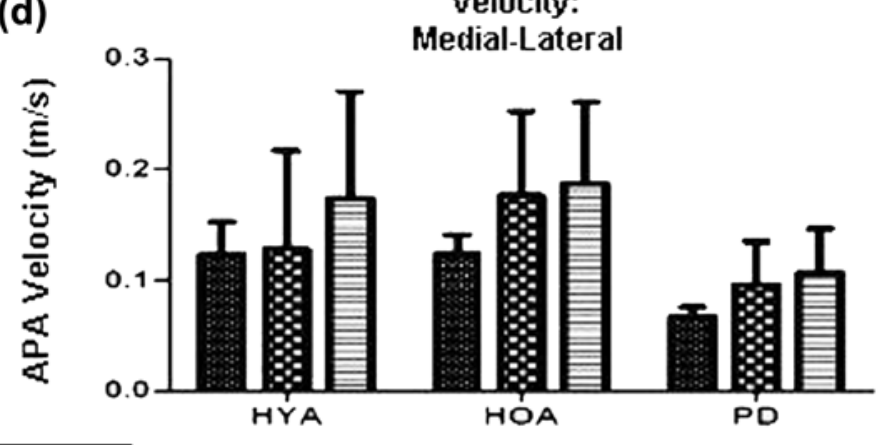

Single
0-Back
2-Back

Figure 2.

Mean and standard error values across groups (healthy young adults [HYA], healthy older adults [HOA], and individuals with Parkinson disease $[P D]$ ) for single-task gait initiation (GI), 0-back GI, and 2-back $\mathrm{Gl}$ on anticipatory postural adjustments (APA) displacement during $\mathrm{GI}$ in (a) anterior-posterior (AP) and (b) medial-lateral (ML) directions and on APA velocity during GI in (c) AP and (d) ML directions. Note that among the four outcome variables, no significant effect of dual task was observed within any group.

required to propel the body forward to initiate forward locomotion. In the present study, during both the singleand dual-task GI paradigms, those with PD had reduced posterior center of pressure displacements and velocities when compared with their aged-matched healthy peers and HYA. Previous studies [16-18] have identified several distinguishing postural abnormalities in PD that may contribute to these findings, including (1) abnormally sized automatic postural responses, particularly enlarged "medium latency" stretch responses in lower-leg muscles; (2) delayed initiation or reduced scaling of voluntary postural responses; and (3) abnormal execution of compensatory stepping movements. Collectively, these decrements compromise propulsive force generation and the required forward momentum production for safe, efficient GI.
Contrary to our hypothesis, the addition of the secondary cognitive task during GI did not disrupt the APA when compared with single-task GI within the PD group. This finding is quite surprising in the PD group, because the detrimental impact of secondary cognitive tasks on previously examined motor tasks, such as standing balance and gait, is robust [19]. It has been hypothesized that the reduction in gait performance and arrhythmicity of gait under a dualtask condition is due to individuals with PD recruiting attentional resources for gait, specifically the frontal-cortical regions, to compensate for the damaged automaticity of gait [20]. Because of limited cognitive resources and the neurodegenerative nature of the disease, the ability to recruit attentional resources is limited, particularly during a dual task and, as such, performance suffers. Thus, it is 


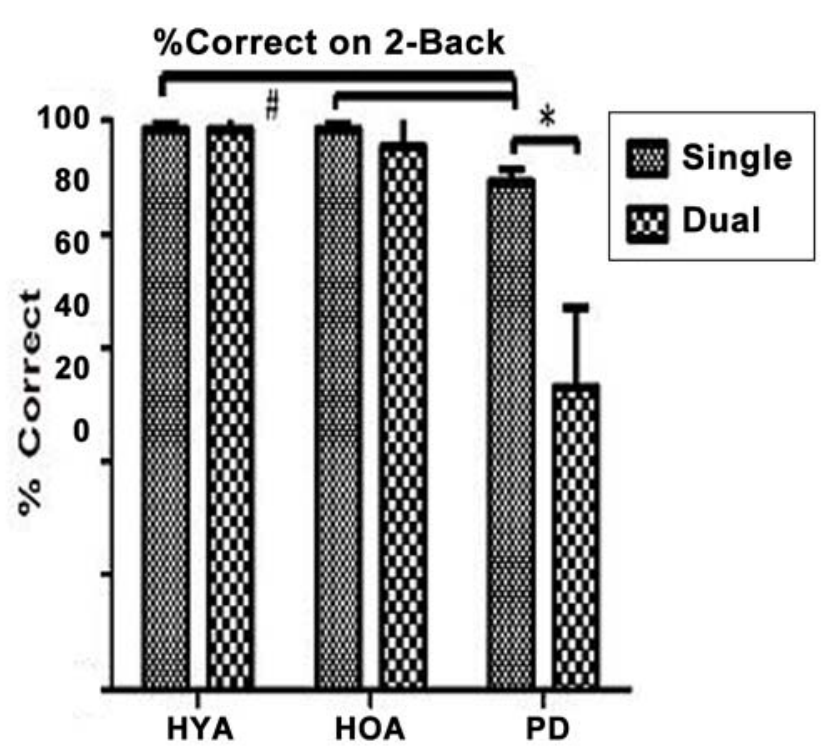

Figure 3.

Percent correct on 2-back during both single- and dual-task settings for healthy young adults (HYA), healthy older adults (HOA), and individuals with Parkinson disease (PD). ${ }^{*}$ Significant difference in percent correct of both HYA and HOA when compared with individuals with PD $(p<0.05)$ during single-task 2-back. *Significant decrease $(p<0.05)$ in percent correct in dual-task setting when compared with single-task setting in PD group. Percent correct during dual-task 2-back in PD (53\%) was significantly equal to chance $(50 \%)$ at 0.05 .

surprising that the APA phase of GI remains unaffected despite the disruptions in gait/balance during a dual task.

An examination of the finding that GI performance was unaffected by a secondary cognitive task is interesting when examined in the context of the "posture second” strategy proposed by Bloem and colleagues [19]. The posture second strategy suggests that PD patients treat all elements of a dual task with equal priority. The authors speculated that this strategy is adopted at the expense of maintaining a safe gait and, as such, may lead to a fall. Importantly, the posture second strategy is proposed in the context of walking/balance tasks. While walking/balance tasks are traditionally considered "automatic" processes regulated by the basal ganglia and/or spinal cord, GI is considered a more voluntary, "intentional” process controlled by higher cortical networks, specifically the juxtapositional lobule cortex. Therefore, our results suggest that during more intentional tasks, like GI, patients with PD may be able to prioritize if they perceive one task as having greater importance in terms of safety and/or self-fulfillment. However, further research on dual-tasking GI is needed to partition out the effect of prioritization.

An additional or perhaps alternative explanation for the lack of dual-task effects during GI in the persons with PD stems from the postulation that GI is indeed more reliant than gait on higher cortical function (juxtapositional lobule cortex). Importantly, clinical findings implicate juxtapositional lobule cortex pathology as a cause of disturbances in GI as well as freezing of GI in PD [11,21]. Further, imaging studies have demonstrated impaired movement-related activation of the juxtapositional lobule cortex in PD [22]. The 2-back, on the other hand, is known to place demands on the higher executive processes, specifically activation in the dorsolateral prefrontal cortex [15]. And much like the juxtapositional lobule cortex, imaging studies have demonstrated compromised dorsolateral prefrontal cortex circuitry during the performance of various working memory tasks in PD [23]. As such, the lower levels of cortical activation in the dorsolateral prefrontal cortex and the juxtapositional lobule cortex during GI in conjunction with a high-load cognitive task (2-back) may result in "limited sharing" of resources and, consequently, a hindrance of performance on one or both of the tasks.

The hypothesis that limited higher cognitive sharing between two higher cognitive requirements is in line with the capacity-sharing model proposed by O'Shea and colleagues [24]. This model suggests that performance on two tasks reduces functioning of one or both tasks if capacity is exceeded, regardless of the nature of the tasks. However, we suggest that the types of tasks involved are crucial. For example, if two tasks (i.e., frontally mediated intentional tasks) require similar resources and those resources are limited, patients may not have the capacity to allot sufficient resources to both tasks to maintain an adequate level of performance. Importantly, the task perceived to be of most importance may benefit from this limited-sharing phenomenon (e.g., GI), while the perceived less important task suffers, as did 2-back performance here. Future research is needed to examine allocation of resources during dual tasking in PD.

While we believe this study does have merits, the limitations should be pointed out. For example, we found no overt cognitive impairment as measured by the MMSE; however, a more sensitive measure of cognitive functioning, particularly to frontally based tasks, could be utilized 
in future studies to assess the effect of cognitive status on GI during a dual task. Utilizing a dual-task paradigm, Rochester and colleagues did demonstrate that decreased executive function increased difficultly in walking for PD [25]. Thus, a more robust evaluation of executive function could provide greater insight into mediating factors contributing to decreased dual-task performance in PD.

\section{CONCLUSIONS}

In conclusion, the results of this study confirm that patients with PD exhibited less APA than aged-matched HOA and HYA during GI. Of interest was the finding of no additional effect on APA of GI during dual-task conditions in any group, including the PD group. Future research is needed to partition out the effects of prioritization and allocation of resources.

\section{ACKNOWLEDGMENTS}

\author{
Author Contributions: \\ Study concept and design: J. R. Nocera, L. J. P. Altmann, C. J. Hass. \\ Acquisition of data: J. R. Nocera, R. Roemmich, J. Elrod. \\ Analysis and interpretation of data: J. R. Nocera, R. Roemmich, \\ J. Elrod, C. J. Hass. \\ Drafting of manuscript: J. R. Nocera. \\ Critical revision of manuscript for important intellectual content: \\ R. Roemmich, L. J. P. Altmann. \\ Statistical analysis: J. R. Nocera. \\ Study supervision: C. J. Hass.
}

Financial Disclosures: The authors have declared that no competing interests exist.

Funding/Support: This material was based on work supported by the National Institute on Deafness and Other Communication Disorders (grant T32DC008768) and the Department of Veterans Affairs (grant E6860M).

Institutional Review: All participants read and provided informed consent as approved by the University of Florida Institutional Review Board.

Participant Follow-Up: The authors do not plan to inform participants of the publication of this study. However, participants have been encouraged to check the study Web site for updated publications.

\section{REFERENCES}

1. Hass CJ, Waddell DE, Fleming RP, Juncos JL, Gregor RJ. Gait initiation and dynamic balance control in Parkinson's disease. Arch Phys Med Rehabil. 2005;86(11):2172-76.

\section{[PMID:16271566]}

http://dx.doi.org/10.1016/j.apmr.2005.05.013

2. Hass CJ, Waddell DE, Wolf SL, Juncos JL, Gregor RJ. Gait initiation in older adults with postural instability. Clin Biomech (Bristol, Avon). 2008;23(6):743-53.

[PMID:18407387]

http://dx.doi.org/10.1016/j.clinbiomech.2008.02.012

3. Roemmich RT, Nocera JR, Vallabhajosula S, Amano S, Naugle KM, Stegemöller EL, Hass CJ. Spatiotemporal variability during gait initiation in Parkinson's disease. Gait Posture. 2012;36(3):340-43. [PMID:22543093] http://dx.doi.org/10.1016/j.gaitpost.2012.01.018

4. Alves G, Larsen JP, Emre M, Wentzel-Larsen T, Aarsland D. Changes in motor subtype and risk for incident dementia in Parkinson's disease. Mov Disord. 2006;21(8):1123-30. [PMID:16637023] http://dx.doi.org/10.1002/mds.20897

5. Nocera JR, Price C, Fernandez HH, Amano S, Vallabhajosula S, Okun MS, Hwynn N, Hass CJ. Tests of dorsolateral frontal function correlate with objective tests of postural stability in early to moderate stage Parkinson's disease. Parkinsonism Relat Disord. 2010;16(9):590-94. [PMID:20829093] http://dx.doi.org/10.1016/j.parkreldis.2010.08.008

6. Woollacott M, Shumway-Cook A. Attention and the control of posture and gait: a review of an emerging area of research. Gait Posture. 2002;16(1):1-14.

[PMID:12127181] http://dx.doi.org/10.1016/S0966-6362(01)00156-4

7. Hausdorff JM, Balash J, Giladi N. Effects of cognitive challenge on gait variability in patients with Parkinson's disease. J Geriatr Psychiatry Neurol. 2003;16(1):53-58. [PMID:12641374]

8. Marchese R, Bove M, Abbruzzese G. Effect of cognitive and motor tasks on postural stability in Parkinson's disease: a posturographic study. Mov Disord. 2003;18(6):652-58. [PMID:12784268] http://dx.doi.org/10.1002/mds.10418

9. Melzer I, Oddsson LI. The effect of a cognitive task on voluntary step execution in healthy elderly and young individuals. J Am Geriatr Soc. 2004;52(8):1255-62.

[PMID:15271111] http://dx.doi.org/10.1111/j.1532-5415.2004.52353.x

10. Mancini M, Zampieri C, Carlson-Kuhta P, Chiari L, Horak FB. Anticipatory postural adjustments prior to step initiation are hypometric in untreated Parkinson's disease: an accelerometer-based approach. Eur J Neurol. 2009;16(9): 1028-34. [PMID:19473350] http://dx.doi.org/10.1111/j.1468-1331.2009.02641.x

11. Nadeau SE. Gait apraxia: further clues to localization. Eur Neurol. 2007;58(3):142-45. [PMID:17622719] http://dx.doi.org/10.1159/000104714 
12. Stollstorff M, Foss-Feig J, Cook EH Jr, Stein MA, Gaillard WD, Vaidya CJ. Neural response to working memory load varies by dopamine transporter genotype in children. Neuroimage. 2010;53(3):970-77. [PMID:20053379] http://dx.doi.org/10.1016/j.neuroimage.2009.12.104

13. Jaeggi SM, Seewer R, Nirkko AC, Eckstein D, Schroth G, Groner R, Gutbrod K. Does excessive memory load attenuate activation in the prefrontal cortex? Load-dependent processing in single and dual tasks: functional magnetic resonance imaging study. Neuroimage. 2003;19(2 Pt 1):210-25. [PMID:12814572] http://dx.doi.org/10.1016/S1053-8119(03)00098-3

14. Tamura I, Kikuchi S, Otsuki M, Kitagawa M, Tashiro K. Deficits of working memory during mental calculation in patients with Parkinson's disease. J Neurol Sci. 2003; 209(1-2):19-23. [PMID:12686397] http://dx.doi.org/10.1016/S0022-510X(02)00457-4

15. Alberts JL, Voelcker-Rehage C, Hallahan K, Vitek M, Bamzai R, Vitek JL. Bilateral subthalamic stimulation impairs cognitive-motor performance in Parkinson's disease patients. Brain. 2008;131(Pt 12):3348-60.

[PMID:18842609]

http://dx.doi.org/10.1093/brain/awn238

16. Chong RK, Horak FB, Woollacott MH. Parkinson's disease impairs the ability to change set quickly. J Neurol Sci. 2000;175(1):57-70. [PMID:10785258] http://dx.doi.org/10.1016/S0022-510X(00)00277-X

17. Horak FB, Frank J, Nutt J. Effects of dopamine on postural control in parkinsonian subjects: scaling, set, and tone. J Neurophysiol. 1996;75(6):2380-96. [PMID:8793751]

18. Horak FB, Nutt JG, Nashner LM. Postural inflexibility in parkinsonian subjects. J Neurol Sci. 1992;111(1):46-58. [PMID:1402997] http://dx.doi.org/10.1016/0022-510X(92)90111-W

19. Bloem BR, Grimbergen YA, van Dijk JG, Munneke M. The "posture second" strategy: a review of wrong priorities in Parkinson's disease. J Neurol Sci. 2006;248(1-2):196-204. [PMID:16806270] http://dx.doi.org/10.1016/j.jns.2006.05.010

20. Morris ME, Iansek R, Matyas TA, Summers JJ. Stride length regulation in Parkinson's disease. Normalization strategies and underlying mechanisms. Brain. 1996;119(Pt 2):551-68. [PMID:8800948] http://dx.doi.org/10.1093/brain/119.2.551

21. Reetz K, Siebner HR, Gaser C, Hagenah J, Buechel C, Kasten M, Petersen D, Pramstaller PP, Klein C, Binkofksi
F. Premotor gray matter volume is associated with clinical findings in idiopathic and genetically determined Parkinson's disease. Open Neuroimag J. 2008;2:102-5.

[PMID:19526072]

http://dx.doi.org/10.2174/1874440000802010102

22. Jacobs JV, Lou JS, Kraakevik JA, Horak FB. The supplementary motor area contributes to the timing of the anticipatory postural adjustment during step initiation in participants with and without Parkinson's disease. Neuroscience. 2009;164(2):877-85. [PMID:19665521] http://dx.doi.org/10.1016/j.neuroscience.2009.08.002

23. Zgaljardic DJ, Borod JC, Foldi NS, Mattis PJ, Gordon MF, Feigin A, Eidelberg D. An examination of executive dysfunction associated with frontostriatal circuitry in Parkinson's disease. J Clin Exp Neuropsychol. 2006;28(7):1127-44. [PMID:16840240] http://dx.doi.org/10.1080/13803390500246910

24. O’Shea S, Morris ME, Iansek R. Dual task interference during gait in people with Parkinson disease: effects of motor versus cognitive secondary tasks. Phys Ther. 2002; 82(9):888-97. [PMID:12201803]

25. Rochester L, Hetherington V, Jones D, Nieuwboer A, Willems AM, Kwakkel G, Van Wegen E. Attending to the task: interference effects of functional tasks on walking in Parkinson's disease and the roles of cognition, depression, fatigue, and balance. Arch Phys Med Rehabil. 2004;85(10): 1578-85. [PMID:15468014] http://dx.doi.org/10.1016/j.apmr.2004.01.025

Submitted for publication June 19, 2012. Accepted in revised form November 14, 2012.

This article and any supplementary material should be cited as follows:

Nocera JR, Roemmich R, Elrod J, Altmann LJ, Hass CJ. Effects of cognitive task on gait initiation in Parkinson disease: Evidence of motor prioritization? J Rehabil Res Dev. 2013;50(5):699-708.

http://dx.doi.org/10.1682/JRRD.2012.06.0114

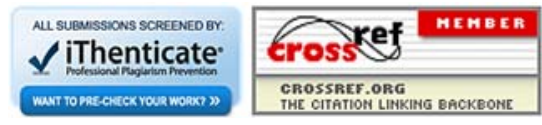


